\title{
Cardiac Rehabilitation to Improve Physical Functioning in Refractory Angina: A Pilot Study
}

\author{
Elizabeth A. Asbury ${ }^{a, b}$ Carolyn M. Webb ${ }^{a, b}$ Heather Probert ${ }^{c}$ Christine Wright $^{b}$ \\ Mahmoud Barbirc Kim Fox ${ }^{b}$ Peter Collins ${ }^{a, b}$ \\ ${ }^{a}$ Cardiovascular Sciences, National Heart and Lung Institute, Imperial College London, ${ }^{\mathrm{b}}$ Institute of Cardiovascular \\ Medicine and Science, Royal Brompton Hospital, and ${ }^{\mathrm{C} C a r d i a c ~ H e a l t h ~ P r o m o t i o n, ~ H a r e f i e l d ~ H o s p i t a l, ~ R o y a l ~ B r o m p t o n ~}$ \\ and Harefield NHS Foundation Trust, London, UK
}

For editorial comment see p. 167

\section{Key Words}

Refractory angina $\cdot$ Cardiac rehabilitation $\cdot$ Psychological morbidity $\cdot$ Quality of life

\begin{abstract}
Objectives: Refractory angina patients suffer debilitating chest pain despite optimal medical therapy and previous cardiovascular intervention. Cardiac rehabilitation is often not prescribed due to a lack of evidence regarding potential efficacy and patient suitability. A randomised controlled study was undertaken to explore the impact of cardiac rehabilitation on cardiovascular risk factors, physical ability, quality of life and psychological morbidity among refractory angina sufferers. Methods: Forty-two refractory angina patients $(65.1 \pm 7.3$ years) were randomly assigned to an 8-week Phase III cardiac rehabilitation program or symptom diary control. Physical assessment, Progressive Shuttle Walk test, Hospital Anxiety and Depression Scale, Health Anxiety Questionnaire, the York Angina Beliefs scale, ENRICHD Social Support Instrument and SF-36 were completed before and after intervention and at 8-week follow-up. Results: Following cardiac rehabilitation, patients demonstrated improved physical ability compared with controls in Progres-
\end{abstract}

sive Shuttle Walk level attainment $(p=0.005)$ and total distance covered $(p=0.015)$. Angina frequency and severity remained unchanged in both groups, with the control demonstrating worsening SF-36 pain scale $(63.43 \pm 22.28$ vs. $55.46 \pm 23.98, p=0.025)$. Cardiac rehabilitation participants showed improved Health Anxiety Questionnaire reassurance (1.71 \pm 1.72 vs. $1.14 \pm 1.23, p=0.026)$ and York Beliefs anginal threat perception (12.42 \pm 4.58 vs. $14.35 \pm 4.73$, $\mathrm{p}=0.05$ ) after cardiac rehabilitation. Physical measures were broadly unaffected. Conclusions: Cardiac rehabilitation can be prescribed to improve physical ability without affecting angina frequency or severity among patients with refractory angina.

Copyright $\odot 2012$ S. Karger AG, Basel

\section{Introduction}

The majority of patients presenting with angina pectoris resulting from coronary heart disease (CHD) are successfully treated with interventions including coronary artery bypass grafting (CABG) and percutaneous coronary intervention (PCI) and medical management [1]. However, a growing number of patients experience

\section{KARGER}

Fax +41613061234

E-Mail karger@karger.ch

www.karger.com (c) 2012 S. Karger AG, Basel

$0008-6312 / 12 / 1223-0170 \$ 38.00 / 0$

Accessible online at:

www.karger.com/crd
Elizabeth Asbury

Cardiovascular Sciences, National Heart and Lung Institute

Imperial College London, Dovehouse Street

London SW3 6LY (UK)

Tel. +64 4905 3262, E-Mail asbury.elizabeth@gmail.com 
persistent, refractory angina in spite of intervention and optimal medical treatment $[2,3]$. Current estimates suggest as many as $10 \%$ of all patients diagnosed with $\mathrm{CHD}$ will develop refractory angina, highlighting an ever increasing population of patients with a symptomatic untreatable chronic disease [1].

Previous studies have explored the effect of various therapeutic interventions, including stellate ganglion blocks [4], electrical neuromodulation devices, such as transcutaneous electrical nerve stimulation [5] and spinal cord stimulation [6,7], enhanced external counterpulsation [8], percutaneous myocardial laser revascularisation [9] and a specifically designed management program [10]. However, such treatments have failed to demonstrate a universal impact on the chest pain and dyspnoea associated with refractory angina.

In accordance with studies of patients with $\mathrm{CHD}$, refractory angina is associated with increased psychological morbidity and a chronically impaired quality of life [10]. It has been suggested that interventions to reduce psychological distress have the potential to improve longterm cardiac prognosis [11]. Cardiac rehabilitation has been shown to improve depression [12], anxiety [13], and quality of life among patients with CHD [14]; however, as many as $50 \%$ of patients undergoing cardiac rehabilitation following myocardial infarction (MI) suffer from clinically high levels of psychological distress [15]. A combined treatment package of outpatient counselling, cardiac rehabilitation and cognitive behavioural therapy has been shown to improve psychological morbidity and quality of life among refractory angina patients [16]. However, studies exploring the physiological and psychological impact of routinely available cardiac rehabilitation as a stand-alone intervention for refractory angina have not previously been undertaken.

The updated American Heart Association recommendations for exercise and training [17] advocates cardiac rehabilitation and supervised exercise training for patients with ongoing angina, previous history of CABG, MI, PCI and patients with existing cardiomyopathy. Meta-analysis has shown that exercise-based rehabilitation does not increase the risk of non-fatal MI, revascularisation, cardiac or all-cause mortality among existing CHD cardiac rehabilitation participants [18]. Cardiac rehabilitation has been recommended as a suitable treatment for refractory angina for improving myocardial perfusion and quality of life [2], along with long-term prognosis and functional capacity [1]. Nevertheless, cardiac rehabilitation centres are reluctant to accept patients with ongoing angina or complicated cardiovascular history in spite of the duration or stability of the symptoms. Indeed, angina and heart failure are often used to exclude patients from cardiac rehabilitation [19]. It has been argued that cardiac rehabilitation should be made more widely available, as the benefits of cardiovascular exercise therapy should not be limited to the refined subgroup of cardiovascular patients currently admitted to cardiac rehabilitation [18]. However, the paucity of data and a lack of empirical research exploring the impact of exercise therapy among patients with refractory angina are prohibiting patients from participating in a potentially beneficial treatment program.

Therefore, this study was designed to establish whether an 8-week cardiac rehabilitation exercise program is an appropriate treatment option for patients with refractory angina. The primary end point was effect of cardiac rehabilitation on anxiety and depression, with secondary end points including physical and physiological functioning, symptom frequency and severity, and other measures of psychological morbidity and quality of life.

\section{Methods}

The study was designed as a randomised control trial and was approved by the Royal Brompton \& Harefield NHS Foundation Trust Ethics Committee. All patients gave written informed consent. The use of group blinding was expressly forbidden by the local ethics committee due to issues relating to obtaining fully informed consent. Patients with the full diagnostic criteria for refractory angina who fulfilled the study inclusion criteria (table 1) were recruited from cardiology outpatients, pain and specialised angina clinics at the Royal Brompton \& Harefield NHS Foundation Trust. Eligible patients were also identified from the recent cardiac catheterisation lists and approached to participate by mail.

Following consent, patients were randomly assigned to complete either an 8-week cardiac rehabilitation and symptom monitoring program or 8-week symptom monitoring only control in addition to standard treatment. Randomisation was performed using 60 identical opaque brown sealed envelopes containing an equal number of paper strips marked 'Rehabilitation' or 'Monitoring'. Physical measurements were taken, including resting blood pressure, height, weight, fasting blood sample (lipid profile, glucose, haemoglobin, U\&E, LFT, TFT), resting 12-lead ECG and a telemetry-monitored Progressive Shuttle Walk (PSW) exercise tolerance test [20]. All patients were then guided through and asked to complete a number of questionnaires (see the section 'Questionnaires' below), while the symptom diary was explained in detail. Patients randomised to the control arm were requested to start the symptom monitoring diary immediately, and to desist from any new self-motivated, independent exercise program while participating in the study. Adherence to this request was checked at each subsequent study visit. Patients randomised to cardiac rehabilitation were instructed to begin the diary the week of their first class. 
Table 1. Inclusion and exclusion criteria for study participants

\begin{tabular}{|c|c|}
\hline Inclusion criteria & Exclusion criteria \\
\hline Clinically diagnosed with refractory angina $>6$ months & History of any other chronic illness \\
\hline Two or more episodes of angina per week & $\begin{array}{l}\text { Participation in two or more periods of planned moderate } \\
\text { intensity exercise per week during the past } 6 \text { months }\end{array}$ \\
\hline Males and females aged $30-80$ years & $\begin{array}{l}\text { Suffer any physical condition for which exercise is a } \\
\text { contra-indication }\end{array}$ \\
\hline $\begin{array}{l}\text { Previous history of single or multiple MI, CABG, PTCA or any } \\
\text { combination of the above }\end{array}$ & $\begin{array}{l}\text { Participation in another research study within the previous } \\
60 \text { days }\end{array}$ \\
\hline $\begin{array}{l}\text { Prescribed optimal medical therapy and not consideredsuitable } \\
\text { for further revascularisation }\end{array}$ & History of psychiatric illness \\
\hline AHA exercise and training guidelines classification B and C & AHA exercise and training guidelines classification class D \\
\hline Willing to give written informed consent & Unwilling to give written informed consent \\
\hline
\end{tabular}

Following the 8 -week intervention, physical measurements and PSW tests were repeated, while patients returned the symptom monitoring diaries and completed a further set of questionnaires, identical to those completed at baseline, with supplementary questions relating to changes in medication, illnesses or hospitalisations during the 8-week intervention. The physical measurements, PSW test and questionnaires were completed a final time by all patients after a further 8-week follow-up period, where additional questions were asked relating to exercise participation and physical activity levels.

\section{Questionnaires}

The Health Anxiety Questionnaire (HAQ) [21] is comprised of four subscales, which measure health worry and preoccupation, fear of illness and death, reassurance-seeking behaviour and interference with life. The Hospital Anxiety and Depression Scale (HADS) [22] measures clinically significant anxiety and depression in general medical patients, while the SF-36 [23] is a general health questionnaire divided into different aspects of quality of life: physical functioning, physical role, bodily pain, general health, vitality, social functioning, emotional role and mental health. The York Angina Beliefs scale [24] is designed to uncover the patient's beliefs and possible misconceptions regarding chest pain symptoms and dyspnoea. Finally, patients were asked to complete the ENRICHD Social Support Instrument [25], which assesses four key attributes of social support: emotional, instrumental, informational and appraisal. Patients were also asked questions relating to symptom severity and frequency, along with the year of diagnosis and the duration of suffering.

\section{Cardiac Rehabilitation}

The cardiac rehabilitation intervention was comprised of a standard 8-week group-based Phase III cardiac rehabilitation exercise program, which conformed to the British Association for
Cardiac Rehabilitation guidelines [26]. The outpatient cardiovascular exercise program was designed to improve aerobic conditioning, functional capacity, muscular strength, endurance and flexibility. Participants were encouraged to attend the weekly health promotion seminars, covering topics including stress management, medication, physical activity and dietary advice, although due to the regional variation across current cardiac rehabilitation centres, this was not a mandatory component of the study. Study patients were amalgamated into existing classes (routine procedure due to the constant patient turnover in cardiac rehabilitation groups) and allocated on the basis of physical ability rather than diagnosis. Each cardiac rehabilitation class was approximately $80 \mathrm{~min}$ in duration, with patients moving through a circuit of activities, alternating between cardiovascular and active rest stations. The aim was for cardiac rehabilitation participants with normal left ventricular function to exercise at $60-75 \%$ of their age-predicted heart rate reserve, with a target of $40-60 \%$ if the patient presented with an ejection fraction of less than $40 \%$.

The supporting home exercise program comprised of activities such as brisk walking, along with movement and strengthening exercises. Patients were requested to perform a minimum of one home-based cardiac rehabilitation session per week, while also improving their daily activity levels. Patients recorded their weekly home-based exercise participation, which was reviewed during each hospital-based cardiac rehabilitation session.

\section{Symptom Monitoring}

Both the cardiac rehabilitation and control groups were required to complete a symptom monitoring diary for the duration of the 8-week intervention period. All participants were asked to complete the diary each day, noting the symptoms they associated with their angina, severity of the attack, and activity and time of day of onset. Patients were instructed to grade their symptoms using a standard five-point pain scale. 
Table 2. Risk factors and cardiovascular history of the entire patient cohort

\begin{tabular}{lc}
\hline Risk factors and cardiovascular history & Percentage of cohort \\
\hline MI & 66.7 \\
CABG & 69.0 \\
PCI & 64.3 \\
Valve replacement & 4.8 \\
Pacemaker & 7.1 \\
Family history of CHD & 50.0 \\
Diabetes & 35.7 \\
Hypertension & 59.5 \\
Hypercholesterolaemia & 76.2 \\
Smoking history & 57.1 \\
\hline
\end{tabular}

Statistics and Sample Size Calculation

The study was designed as randomised within- and betweensubjects design, comparing active with control and changes within both groups on each measure at all three study time points, using two-tailed tests. The data were analysed using one-way ANOVA, paired and independent Students' $t$ tests and ANCOVA to determine between-group effects while controlling for baseline variation; overall comparisons of categorical data were performed using $\chi^{2}$ test. Statistical significance level was set at $p<0.05$. Data are expressed as mean \pm standard deviation. In order to ensure a minimum $80 \%$ power with a $5 \%$ significance level, the mean group differences of each HADS and HAQ subscale from a previous cross-sectional study [27] (HADS depression: $2.1 \pm 3.5, \mathrm{n}=$ 85; HAQ interference with life: $1.9 \pm 2.4, \mathrm{n}=50$; total HAQ: 5.6 $\pm 7.8, \mathrm{n}=65$ ) were used to establish the average sample size required using the standard calculation and normogram [28]. Using these parameters, it was calculated that a sample size of 70 would enable the accurate assessment of cardiac rehabilitation as a treatment for patients with refractory angina.

\section{Results}

Forty-two patients with refractory angina $(65.1 \pm 7.3$ years) with an average cardiovascular history of 12.3 $( \pm 8.7)$ years were consented into the study. Twenty-two patients $(65.1 \pm 7.1$ years) were randomised to cardiac rehabilitation and $20(65.1 \pm 7.7$ years, $\mathrm{p}=0.98)$ to the control. The risk factors and cardiovascular history of the patient cohort are shown in table 2. There were no significant differences in the cardiovascular medications (statins, aspirin, beta-blockers, diuretics, ACE inhibitors, calcium channel blockers, nitrates, potassium channel activators, antiplatelet therapy and GTN) used by both groups (table 3). Seventeen percent of patients were female and equally represented in both groups. Two pa-
Table 3. Number of patients prescribed cardiovascular medications in each group

\begin{tabular}{lccl}
\hline & $\begin{array}{l}\text { Rehab group } \\
(\mathrm{n}=20)\end{array}$ & $\begin{array}{l}\text { Control group } \\
(\mathrm{n}=20)\end{array}$ & $\mathrm{p}$ \\
\hline Statin & 20 & 19 & 1.000 \\
Aspirin & 15 & 17 & 0.695 \\
Beta-blockers & 14 & 13 & 1.000 \\
Diuretic & 4 & 12 & 0.086 \\
Calcium channel blockers & 4 & 5 & 1.000 \\
Potassium channel activators & 8 & 8 & 1.000 \\
ACE inhibitors & 16 & 12 & 0.301 \\
GTN & 9 & 12 & 0.537 \\
Antiplatelet therapy & 6 & 10 & 0.333 \\
Nitrates & 7 & 9 & 0.748 \\
\hline
\end{tabular}

tients randomised to cardiac rehabilitation withdrew following randomisation (family problems, comorbidities) while a further patient was withdrawn from the study after suffering a non-ST-elevation MI and cerebrovascular accident following randomisation but prior to starting cardiac rehabilitation. Two patients participating in the rehabilitation program suffered medical complications during the exercise classes (panic attack, vasovagal following coughing fit related to previously undiagnosed bronchiectasis) and were admitted for observation. Both were discharged without event and completed the study. Therefore, 19 patients randomised to cardiac rehabilitation successfully completed the 8-week cardiac rehabilitation program completing an average of seven (range 3-12) classes, along with comparable number of home exercise sessions (range 2-12). Twenty patients randomised to the symptom monitoring control completed the study.

\section{Physical Ability}

There were no baseline differences between the groups on either measure of physical ability (table 4). Nevertheless, when controlling for baseline variation, there was a significant difference between the groups' PSW level attainment $(\mathrm{p}=0.005)$, with cardiac rehabilitation participants $(4.19 \pm 1.15$ vs. $5.44 \pm 1.50, \mathrm{p}<0.001)$ showing greater improvements than controls $(4.04 \pm 1.3$ vs. 4.50 $\pm 1.2, \mathrm{p}=0.002)$. This is also reflected in the PSW distance covered, with a significant difference between the groups $(\mathrm{p}=0.015)$ and within the groups at each time point (fig. 1). 


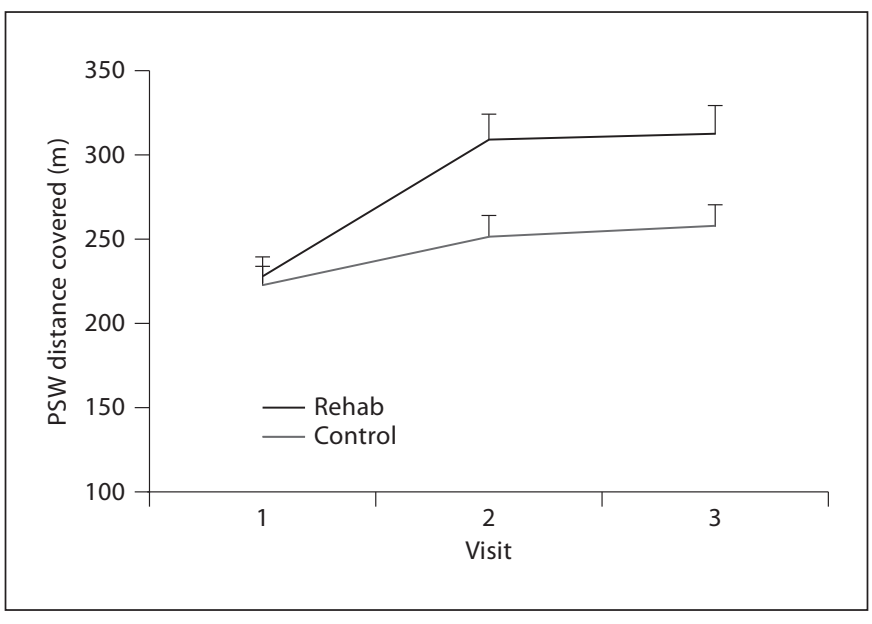

Fig. 1. Difference in the PSW test distance covered between the study groups at all three time points in the study.

\section{Angina Frequency and Severity}

The angina diaries showed no baseline differences between groups on either angina frequency or severity (table 4). This continued throughout the study, and while a trend towards reduced angina severity was noted within the rehab group $(1.82 \pm 0.88$ vs. $1.21 \pm 1.28, \mathrm{p}=0.07)$, no significant differences were found between or within either group at any time point.

\section{Physiological Measures}

Patients randomised to the control group had a higher waist/hip ratio, heart rate and fasting plasma glucose at baseline than the cardiac rehabilitation group (table 4), but there were no other significant differences between the groups on physiological measures at baseline. Both the cardiac rehabilitation group (74.62 \pm 9.76 vs. 69.25 $\pm 8.19, \mathrm{p}=0.037)$ and control $(75.12 \pm 11.72$ vs. 70.57 $\pm 9.97, \mathrm{p}=0.046$ ) showed broadly similar improvements in diastolic blood pressure following the 8-week intervention/control. This was mirrored by measures of systolic blood pressure, with both cardiac rehabilitation $(133.92 \pm 17.26$ vs. $120.1 \pm 19.9, \mathrm{p}=0.001)$ and control $(130.20 \pm 24.3$ vs. $122.1 \pm 17.43, \mathrm{p}=0.032)$ groups showing a significant decrease. However, no significant difference was maintained at follow-up. With the exception of a trend towards lower LDL measures at follow-up within the cardiac rehabilitation group $(2.38 \pm 0.68$ vs. $2.21 \pm 0.62, \mathrm{p}=0.07)$, there were no other differences within or between the groups on any other physiological measure.
Table 4. Baseline physiological measures, physical ability and angina frequency and severity of the study cohort

\begin{tabular}{lccl}
\hline & $\begin{array}{l}\text { Rehab group } \\
(\mathrm{n}=20)\end{array}$ & $\begin{array}{l}\text { Control group } \\
(\mathrm{n}=20)\end{array}$ & $\mathrm{p}$ \\
\hline Angina frequency/week & $4.85 \pm 4.18$ & $7.84 \pm 5.23$ & 0.096 \\
Angina severity (1-5) & $1.83 \pm 0.88$ & $2.01 \pm 0.66$ & 0.23 \\
Height, cm & $168.8 \pm 6.92$ & $168.1 \pm 8.45$ & 0.75 \\
Weight, kg & $81.2 \pm 10.47$ & $85.1 \pm 18.46$ & 0.41 \\
Waist/hip ratio & $0.97 \pm 0.08$ & $1.02 \pm 0.08$ & 0.02 \\
Heart rate, bpm & $60.0 \pm 11.11$ & $68.1 \pm 11.55$ & 0.028 \\
Haemoglobin, g/dl & $14.39 \pm 1.36$ & $13.84 \pm 1.63$ & 0.25 \\
WBC total, 109/1 & $6.89 \pm 1.93$ & $7.87 \pm 1.54$ & 0.082 \\
Glucose, mmol/l & $5.7 \pm 1.23$ & $7.14 \pm 2.37$ & 0.022 \\
Total cholesterol, mmol/1 & $4.14 \pm 0.81$ & $4.12 \pm 1.12$ & 0.94 \\
LDL, mmol/l & $2.37 \pm 0.64$ & $2.24 \pm 1.02$ & 0.62 \\
HDL, mmol/l & $1.14 \pm 0.31$ & $1.04 \pm 0.27$ & 0.31 \\
Triglycerides, mmol/l & $1.38 \pm 0.73$ & $1.49 \pm 0.97$ & 0.68 \\
HDL/LDL ratio & $3.76 \pm 0.76$ & $4.10 \pm 1.27$ & 0.31 \\
Shuttle level (1-15) & $4.45 \pm 1.68$ & $4.10 \pm 1.25$ & 0.44 \\
Total metres, m & $248.18 \pm 121.7$ & $222.0 \pm 78.4$ & 0.41 \\
Systolic BP, mm Hg & $129.4 \pm 19.4$ & $130.96 \pm 23.4$ & 0.81 \\
Diastolic BP, mm Hg & $74.3 \pm 10.1$ & $75.9 \pm 11.4$ & 0.61
\end{tabular}

Data are expressedas mean \pm SD.

\section{Psychological Wellbeing and Quality of Life}

Control and cardiac rehabilitation groups had statistically similar baseline data from each measure and subscale of psychological wellbeing and quality of life (table 5). Patients randomised to cardiac rehabilitation had significantly improved scores of HAQ reassurance (1.71 \pm 1.72 vs. $1.14 \pm 1.23, \mathrm{p}=0.026)$ and York Beliefs anginal threat perception $(12.42 \pm 4.58$ vs. $14.35 \pm 4.73, \mathrm{p}=0.05)$ after cardiac rehabilitation not shown among the controls. Cardiac rehabilitation participants showed no change in pain, while controls showed a significant increase in SF-36 pain perception scale $(63.43 \pm 22.28$ vs. $55.46 \pm 23.98, \mathrm{p}=0.025$ ) following the 8 weeks of symptom monitoring.

However, controls demonstrated reduced HADS anxiety $(7.25 \pm 4.25$ vs. $6.18 \pm 3.52, \mathrm{p}=0.04)$, total HADS (13.12 \pm 7.09 vs. $11.75 \pm 6.49, \mathrm{p}=0.05)$ and HAQ health worry $(6.94 \pm 4.53$ vs. $5.00 \pm 2.95, \mathrm{p}=0.013)$ at visit two not found within the cardiac rehabilitation group. Controls also showed greater improvements in SF-36 physical health than the cardiac rehabilitation group at visit two while controlling for baseline variation $(\mathrm{p}=0.05)$. 
Table 5. Baseline measures of psychological functioning and quality of life of the study cohort

\begin{tabular}{|c|c|c|c|}
\hline & $\begin{array}{l}\text { Rehab group } \\
(\mathrm{n}=20)\end{array}$ & $\begin{array}{l}\text { Control group } \\
(\mathrm{n}=20)\end{array}$ & $\mathrm{p}$ \\
\hline ESSI & $20.75 \pm 4.57$ & $19.65 \pm 5.00$ & 0.47 \\
\hline \multicolumn{4}{|l|}{ HADS } \\
\hline Anxiety & $9.50 \pm 5.19$ & $8.45 \pm 4.69$ & 0.50 \\
\hline Depression & $5.90 \pm 3.83$ & $6.60 \pm 4.13$ & 0.58 \\
\hline Total & $15.40 \pm 8.06$ & $15.04 \pm 7.57$ & 0.88 \\
\hline \multicolumn{4}{|l|}{ HAQ } \\
\hline Health worry & $7.42 \pm 5.71$ & $7.10 \pm 4.66$ & 0.84 \\
\hline Fear of illness & $6.52 \pm 5.34$ & $7.15 \pm 4.81$ & 0.70 \\
\hline Reassurance & $2.05 \pm 1.84$ & $1.75 \pm 1.25$ & 0.55 \\
\hline Interference & $3.00 \pm 2.42$ & $3.30 \pm 2.65$ & 0.71 \\
\hline Total & $19.0 \pm 13.83$ & $19.3 \pm 11.34$ & 0.94 \\
\hline \multicolumn{4}{|l|}{ Health beliefs } \\
\hline Anginal threat & $13.25 \pm 4.37$ & $13.7 \pm 4.80$ & 0.75 \\
\hline Coping with angina & $12.05 \pm 3.26$ & $10.95 \pm 3.13$ & 0.28 \\
\hline Total & $29.45 \pm 7.54$ & $29.2 \pm 6.06$ & 0.90 \\
\hline \multicolumn{4}{|l|}{ SF-36 } \\
\hline Physical function & $47.5 \pm 25.6$ & $39.2 \pm 25.3$ & 0.32 \\
\hline Physical role limitation & $30.5 \pm 40.7$ & $25.0 \pm 40.5$ & 0.67 \\
\hline Emotion role limitation & $53.7 \pm 44.4$ & $45.0 \pm 46.2$ & 0.55 \\
\hline Energy & $41.1 \pm 21.7$ & $42.5 \pm 27.3$ & 0.86 \\
\hline Emotional wellbeing & $65.3 \pm 23.4$ & $71.4 \pm 20.2$ & 0.38 \\
\hline Social functioning & $75.7 \pm 26.2$ & $64.3 \pm 29.0$ & 0.22 \\
\hline Pain & $50.0 \pm 25.6$ & $58.6 \pm 22.9$ & 0.28 \\
\hline General health & $44.6 \pm 17.7$ & $37.7 \pm 21.4$ & 0.28 \\
\hline
\end{tabular}

Data are expressed as mean $\pm \mathrm{SD}$.

\section{Discussion}

As the number of patients with chronic refractory angina pectoris continues to rise [1], providing patients with capacity to maintain and perform the activities of daily life has become paramount. The results of this pilot study suggest that cardiac rehabilitation can help to fulfil this role. The current pilot study has determined that patients with refractory angina can often successfully participate in an established Phase III cardiac rehabilitation program without serious adverse event. Furthermore, these pilot data suggest that refractory angina patients can significantly improve their physical ability through cardiac rehabilitation without compromising the frequency or severity of existing angina. Global pain perception was unaffected among patients participating in cardiac rehabilitation, highlighting the potential for this modality to improve physical function without aggravating any existing comorbidities. Improving physi- cal function without a concurrent increase in angina frequency may also be related to the improvements in psychological morbidity found among those participating in cardiac rehabilitation. Demonstrating that an existing, routinely available treatment program designed for patients in the early stages of cardiovascular disease could also benefit patients with long-term refractory angina may prove invaluable to both patients and clinicians struggling to manage this difficult, uncompromising condition.

Cardiac rehabilitation has previously been advocated as a treatment for refractory angina [1], although there are very few studies exploring the impact of exercise training among this patient group. In spite of recent guidelines promoting cardiac rehabilitation for the treatment of conditions other than recently diagnosed cardiovascular disease and intervention [17], cardiac rehabilitation has recently been described as 'vastly underutilized' [29]. The continued physical activity required to maintain the benefits of any cardiac rehabilitation program has been identified as a barrier to primary participation and referral [2], while the patient's fear of exposure to risk and increased pain has also been identified as a shortcoming [18]. Participation and adherence rates for routine patients participating in cardiac rehabilitation remains low [30], with women, the elderly and ethnic minorities specifically identified as less likely to take part [31]. However, any bias in participation among certain groups with refractory angina has not yet been established. Further research is required to assess barriers to utilisation of cardiac rehabilitation among patients with refractory angina, and strategies to enhance accessibility and adherence rates.

\section{Study Limitations}

By design, pilot studies are intended primarily to assess the feasibility of performing a definitive evaluation of the study aims and, secondarily and if possible, to provide some evidence favouring the appropriateness of undertaking a definitive study. This pilot study has demonstrated the feasibility of applying cardiac rehabilitation therapy to patients with angina refractory to conventional therapy and suggests that benefit result from such application. The true impact of cardiac rehabilitation in patients with refractory angina now must be tested in a larger, more definitive study. Our results strongly suggest that this is a reasonable and potentially beneficial next step.

The strength of conclusions inferable from this study is limited in part by the relatively small sample size. 
Sample sizes are generally small in pilot studies but, in addition, in this study, the projected sample size was not achieved. Thus, power to test underlying hypotheses was inherently limited, possibly explaining the failure to demonstrate statistically significant differences among subgroups for certain outcomes of interest. Factors limiting recruitment included the relative geographic isolation of the rehabilitation centre, the impact of patients' existing comorbidities and patients' fear of exacerbating existing symptoms by exercise. In addition, failure to demonstrate inter-group differences may have resulted in part from concurrent improvements in physical parameters in both control and treated groups, possibly related to increasing familiarity with the environs over the duration of the study. Similar levels of contact and interaction have been associated with improved psychological morbidity and a number of physical measures among a comparable control [32]. The gender inequality in patient recruitment may reflect sex bias among refractory angina patients or the established non-participation of women in cardiac rehabilitation [29]. Future studies should employ a larger representative patient population to further explore the benefit of cardiac rehabilitation on physical functioning, psychological wellbeing and quality of life among patients with refractory angina.

\section{Conclusions}

The challenge of managing the ever increasing numbers of patients presenting with refractory angina requires existing treatments to be refined, and emerging therapies to be embraced. Increasing life expectancy without enabling patients to improve or maintain their physical ability robs sufferers of the dignity of managing daily life. Cardiac rehabilitation is an established, assessable treatment program familiar to both patients and clinicians alike. This pilot study suggests that the application of this technique may provide a substantial impact on patients' physical ability, without compromising comorbid pain, angina or risk of serious adverse event. A larger, more definitive study now is justified to support the very promising potential of cardiac rehabilitation for patients with refractory angina, providing them the opportunity to reestablish a fulfilling, active life.

\section{Acknowledgements}

The authors would like to acknowledge the help, support and expertise of the Cardiac Rehabilitation Team, Harefield Hospital, The Royal Brompton and Harefield NHS Foundation Trust.

This work was supported by The British Heart Foundation (grant number PG/06/087) and the Victor Philip Dahdaleh Charitable Foundation.

\section{References}

1 Mannheimer C, Camici P, Chester MR, et al: The problem of chronic refractory angina; report from the ESC Joint Study Group on the Treatment of Refractory Angina. Eur Heart J 2002;23:355-370.

$\checkmark 2$ DeJongste MJ, Tio RA, Foreman RD: Chronic therapeutically refractory angina pectoris. Heart 2004;90:225-230.

3 Gowda RM, Khan IA, Punukollu G, Vasavada BC, Nair CK: Treatment of refractory angina pectoris. Int J Cardiol 2005; 101:1-7.

4 Chester M, Hammond C, Leach A: Longterm benefits of stellate ganglion block in severe chronic refractory angina. Pain 2000; 87:103-105.

5 Borjesson M: Visceral chest pain in unstable angina pectoris and effects of transcutaneous electrical nerve stimulation (TENS). A review. Herz 1999;24:114-125.

6 Andrell P, Yu W, Gersbach P, et al: Long-term effects of spinal cord stimulation on angina symptoms and quality of life in patients with refractory angina pectoris - results from the European Angina Registry Link Study (EARL). Heart 2010;96:1132-1136.
7 Taylor RS, De VJ, Buchser E, DeJongste MJ: Spinal cord stimulation in the treatment of refractory angina: systematic review and meta-analysis of randomised controlled trials. BMC Cardiovasc Disord 2009;9:13.

$\checkmark 8$ Fitzgerald CP, Lawson WE, Hui JC, Kennard ED: Enhanced external counterpulsation as initial revascularization treatment for angina refractory to medical therapy. Cardiology 2003;100:129-135.

$\checkmark 9$ McNab D, Khan SN, Sharples LD, et al: An open label, single-centre, randomized trial of spinal cord stimulation vs. percutaneous myocardial laser revascularization in patients with refractory angina pectoris: the SPiRiT trial. Eur Heart J 2006;27:1048-1053.

10 Moore RK, Groves D, Bateson S, et al: Health related quality of life of patients with refractory angina before and one year after enrolment onto a refractory angina program. Eur J Pain 2005;9:305-310.
1 Cossette S, Frasure-Smith N, Lesperance F: Clinical implications of a reduction in psychological distress on cardiac prognosis in patients participating in a psychosocial intervention program. Psychosom Med 2001; 63:257-266.

12 Johnston M, Foulkes J, Johnston DW, Pollard B, Gudmundsdottir H: Impact on patients and partners of inpatient and extended cardiac counseling and rehabilitation: a controlled trial. Psychosom Med 1999;61:225233.

13 Yoshida T, Kohzuki M, Yoshida K, et al: Physical and psychological improvements after phase II cardiac rehabilitation in patients with myocardial infarction. Nurs Health Sci 1999;1:163-170.

14 Marchionni N, Fattirolli F, Fumagalli S, et al: Improved exercise tolerance and quality of life with cardiac rehabilitation of older patients after myocardial infarction: results of a randomized, controlled trial. Circulation 2003; 107:2201-2206.

15 Rafanelli C, Roncuzzi R, Finos L, et al: Psychological assessment in cardiac rehabilitation. Psychother Psychosom 2003;72:343-349. 
16 Moore R, Groves D, Hammond C, Leach A, Chester MR: Temporary sympathectomy in the treatment of chronic refractory angina. J Pain Symptom Manage 2005;30:183-191.

$\checkmark 17$ Fletcher GF, Balady GJ, Amsterdam EA, et al: Exercise standards for testing and training: a statement for healthcare professionals from the American Heart Association. Circulation 2001;104:1694-1740.

18 Taylor RS, Brown A, Ebrahim S, et al: Exercise-based rehabilitation for patients with coronary heart disease: systematic review and meta-analysis of randomized controlled trials. Am J Med 2004;116:682-692.

19 Lewin RJ: Improving quality of life in patients with angina. Heart 1999;82:654-655.

-20 Singh SJ, Morgan MD, Scott S, Walters D, Hardman AE: Development of a shuttle walking test of disability in patients with chronic airways obstruction. Thorax 1992; 47:1019-1024.
21 Lucock MP, Morley S: The Health Anxiety Questionnaire. Br J Health Psychol 1996;1: 137-150.

22 Zigmond AS, Snaith RP: The hospital anxiety and depression scale. Acta Psychiatr Scand 1983;67:361-370.

23 Stewart AL, Hays RD, Ware JE Jr: The MOS short-form general health survey. Reliability and validity in a patient population. Med Care 1988;26:724-735.

24 Furze G, Bull P, Lewin RJ, Thompson DR: Development of the York Angina Beliefs Questionnaire. J Health Psychol 2003;8:307315.

25 Enhancing recovery in coronary heart disease patients (ENRICHD): study design and methods. The ENRICHD investigators. Am Heart J 2000;139:1-9.

26 British Association for Cardiac Rehabilitation. Standards and Core Components for Cardiac Rehabilitation. London, British Association for Cardiac Rehabilitation, 2007.
7 Asbury EA, Creed F, Collins P: Distinct psychosocial differences between women with coronary heart disease and cardiac syndrome X. Eur Heart J 2004;25:16951701.

28 Altman D: Practical Statistics for Medical Research. London, Chapman and Hall, 1991.

29 Wenger NK: Current status of cardiac rehabilitation. J Am Coll Cardiol 2008;51:16191631.

30 Davies P, Taylor F, Beswick A, et al: Promoting patient uptake and adherence in cardiac rehabilitation. Cochrane Database Syst Rev 2010;CD007131.

31 Bethell H, Lewin R, Dalal H: Cardiac rehabilitation in the United Kingdom. Heart 2009;95:271-275.

32 Asbury EA, Slattery C, Grant A, Evans L, Barbir M, Collins P: Cardiac rehabilitation for the treatment of women with chest pain and normal coronary arteries. Menopause 2008;15:454-460. 